\title{
Thermosolutal-convective Instability in Stern's Type Configuration
}

\author{
PARDEEP KUMAR ${ }^{1, *}$, HARI MOHAN ${ }^{2}$ \\ ${ }^{1,2}$ Department of Mathematics, ICDEOL, Himachal Pradesh University, \\ Summerhill, Shimla-171005, INDIA.
}

Abstract:- The thermal-convective instability of a stellar atmosphere in the presence of stable solute gradient in Stern's type configuration is studied in the presence of radiative transfer effect. A criterion for monotonic instability is obtained in terms of the source functions $\mathrm{S}$. The criterion for monotonic instability is found to be unchanged in the presence of radiative transfer and rotation effects. The problem of thermosolutal-convective instability of a hydromagnetic composite medium is also studied to include the frictional effects with neutrals. The criterion derived for monotonic instability in terms of heat-loss function is found to be the same in the presence or absence of the collisional effects.

Key-Words:-Frictional effects, Thermosolutal-convective instability, Radiative transfer effect, Rotation,

Received: February 9, 2021. Revised: June 28, 2021. Accepted: July 10, 2021. Published: July 16, 2021.

\section{Introduction}

Defouw [1] has generalized the Schwarzschild criterion for convection to include departures from adiabatic motion and has shown that a thermally unstable atmosphere is also convectively unstable, irrespective of the atmospheric temperature gradient. The instability in which motions are driven by buoyancy forces of a thermally unstable atmosphere has been termed as "thermal convective instability". Defouw [1] has shown that an inviscid stellar atmosphere is unstable is unstable if

$D=\frac{1}{C_{P}}\left(L_{T}-\rho \alpha L_{\rho}\right)+\kappa k^{2}<0$,

where $L$ is the energy lost minus the energy gained per gram per second (the heat loss function) and $\rho, \alpha, \kappa, k, L_{T}, L_{\rho} \quad$ denote respectively the density, the coefficient of thermal expansion, the coefficient of thermometric conductivity, the wave number of the perturbation, the partial derivative of $L$ with respect to temperature $T$ and the partial derivative of $L$ with respect to density $\rho$, both evaluated in the equilibrium state. In general, the instability due to inequality (1) may be either oscillatory or monotonic. Defouw [1] has also shown that inequality (1) is a sufficient condition for monotonic instability in the presence of magnetic field and rotation on thermal convective instability.

Defouw [1] has also studied the effect of radiative transfer on the thermal-convective instability and has found that the source functions $S$ which lead to thermal-convective instability are those for which $S_{T}-(\rho / T) S_{\rho}<$ 0 , the subscripts on $S$ denote the partial derivatives of $S$ with respect to $T$ and $\rho$ both evaluated in the equilibrium state.

A detailed account of thermal convection, under varying assumptions of hydrodynamics and hydromagnetics, has been given by Chandrasekhar [2]. Veronis [3] has considered the problem of thermohaline convection in a layer of fluid heated from below and subjected to a stable salinity gradient. The thermohaline convection in a horizontal layer of viscous fluid heated from below and salted from above has been studied by Nield [4]. In thermohalineconvective instability problem, buoyancy forces can arise not from density differences due to variations in temperature but also from those due to variations in solute concentrations. The conditions under which convective motions are important in stellar atmospheres are usually far 
removed the consideration of a single component fluid and rigid boundaries and therefore it is desirable to consider one gas component acted on by solute concentration gradient and free boundaries. The thermosolutalconvective instability of a stellar atmosphere in the presence of suspended particles has been studied by Sharma and Singh [5]. The criteria for monotonic instability are derived and are found to hold good also in the presence of uniform rotation and uniform magnetic field on the thermosolutal-convective instability.

A partially ionized plasma represents a state which often exists in the Universe. There are several situations where the interaction between the ionized and neutral gas components becomes important in cosmic physics. As a reasonably simple approximation, the plasma may be idealized as a composite mixture of a hydromagnetic (ionized) component and a neutral component, the two interacting through mutual collisional (frictional) effects. Hans [6] made this simplified approximation and found that these collisions have a stabilizing effect on the Rayleigh-Taylor instability.Marcu and Ballai [7] have studied the thermosolutal linear stability of a composite two-component plasma in the presence of Coriolis forces, finite Larmor radius, taking into account the collisions between neutral and ionized particles. The thermosolutal instability appears due to a material convection (thermosolutal convection) in a two component fluid with different molecular diffusivities which contribute in an opposing sense to the locally vertical density gradient. Natural convection with Soret and radiation effects in a binary fluid saturating a horizontal porous layer under the influence of magnetic field has been investigated by Israel-Cookey and Amos [8]. Sheri et. al [9] has studied the transient radiative reactive thermosolutal magnetohydrodynamic convection in inclined MHD hall generator flow with dissipation and cross diffusion.

In the present paper we consider the thermosolutal-convective instability in Stern's type configuration of a stellar atmosphere (the thermal-convective instability in the presence of stable solute gradient) to include the radiative transfer effect. The effects of radiative transfer and rotation are also studied on the thermosolutal-convective instability. The effects of radiative transfer arise when self absorption is not negligible. As a partially ionized plasma represents a state which often exists in the Universe, the problem of thermosolutalconvective instability of a hydromagnetic composite medium is, therefore, studied to include the frictional effects with neutrals. Theseaspects form the subject matter of the present study.

\section{Description of the Instability and Perturbation Equations}

Consider an infinite horizontal fluid layer of thickness $d$ heated from above and subjected to a stable solute concentration gradient so that the temperatures and solute concentrations at the bottom surface $z=0$ are $T_{0}$ and $C_{0}$ and at the upper surface $z=d$ are $T_{1}$ and $C_{1}$ respectively, $z$-axis being taken as vertical. This layer is acted on by a gravity force $\vec{g}(0,0,-g)$. Then, following the Boussinesq approximation, which states that the inertial effects produced by density variation are negligible in comparison to its gravitational effects i.e. $\rho$ can be taken as constant everywhere in the equations of motion except in the term with external force, consequently the equations governing the motion of the fluid assume the following form:

$$
\begin{aligned}
\frac{\partial \vec{v}}{\partial t}+(\vec{v} \cdot \nabla) \vec{v}= & -\frac{1}{\rho_{0}} \nabla p+v \nabla^{2} \vec{v} \\
& +\vec{g}\left(1+\frac{\delta \rho}{\rho_{0}}\right),
\end{aligned}
$$

$\nabla \cdot \vec{v}=0$,

$\frac{\partial C}{\partial t}+(\vec{v} . \nabla) C=\kappa^{\prime} \nabla^{2} C$,

$\rho=\rho_{0}\left[1-\alpha\left(T-T_{0}\right)+\alpha^{\prime}\left(C-C_{0}\right)\right]$.

The suffix zero refers to values at the reference level $z=0$. Equations (2) - (4) express respectively the conservation of momentum, 
mass and solute mass concentration. Equation (5) represents the equation of state. Here $\rho, p, T, C, \vec{v}(u, v, w), g, \alpha$ and $\alpha^{\prime}$ stand for density, pressure, temperature, solute concentration, velocity, gravitational acceleration, thermal coefficient of expansion and an analogous solvent coefficient, respectively. The kinematic viscosity, the thermal diffusivity $\kappa$ and the solute diffusivity $\kappa^{\prime}$ are each assumed to be constant.

The steady state solution is

$\vec{v}=0, T=T_{0}+\beta z, C=C_{0}-\beta^{\prime} z, \rho=$ $\rho_{0}\left[1-\alpha \beta z-\alpha^{\prime} \beta^{\prime} z^{\prime}\right]$,

where

$$
\beta=\frac{T_{1}-T_{0}}{d} \text {, and } \beta^{\prime}=\frac{C_{0}-C_{1}}{d}
$$

are the magnitudes of uniform temperature and concentration gradients.

We now consider a small perturbation on the steady state solution and let $\vec{v}, \delta \rho, \delta p, \theta$ and $\gamma$ denote the perturbations in velocity, density, pressure, temperature and solute concentration respectively so that the change in density $\delta \rho$, caused by the perturbations $\theta$ and $\gamma$ in temperature and concentration, is given by

$\delta \rho=-\rho_{0}\left(\alpha \theta-\alpha^{\prime} \gamma\right)$.

Equations (2) - (4) on linearization give

$$
\frac{\partial \vec{v}}{\partial t}=-\frac{1}{\rho_{0}} \nabla \delta p+v \nabla^{2} \vec{v}-\vec{g}\left(\alpha \theta-\alpha^{\prime} \gamma\right),
$$

$\nabla \cdot \vec{v}=0$

$\frac{\partial \gamma}{\partial t}-\kappa^{\prime} \nabla^{2} \gamma=\beta^{\prime} w$

Now, the first law of thermodynamics may be written in the form

$$
C_{v} \frac{d T}{d t}=-L+\frac{K}{\rho} \nabla^{2} T+\frac{P}{\rho^{2}} \frac{d \rho}{d t},
$$

where $L, K, C_{v}, T, t$ and $\rho$ denote respectively, the heat-loss function, the thermal conductivity, the specific heat at constant volume, the temperature, the time and the pressure.
Following Defouw [1], the linearized perturbation form of equation (11) is

$$
\begin{array}{r}
\frac{\partial \theta}{\partial t}+\frac{1}{C_{p}}\left(L_{T}-\rho \alpha L_{\rho}\right) \theta-\kappa \nabla^{2} \theta \\
=-\left(\beta+\frac{g}{C_{P}}\right) w .
\end{array}
$$

The effects of radiative transfer arise when selfabsorption is not negligible. In considering the heat-loss function $L(\rho, T)$ to be dependent only on the local values of density and temperature, it is assumed that the gas is optically thin. We shall study radiative transfer with the Eddington approximation which is accurate in both the optically thick and optically thin limits as pointed by Unno and Spiegel [10].

The heat equation for a grey gas, using this approximation, may be written as

$$
\begin{aligned}
\rho C_{v} \frac{d T}{d t}-\frac{p}{\rho} \frac{d \rho}{d t} & =\nabla \cdot\left[\frac { 1 } { 3 m \rho } \nabla \left\{\frac { 1 } { m \rho } \left(\rho C_{v} \frac{d T}{d t}\right.\right.\right. \\
& \left.\left.\left.-\frac{p}{\rho} \frac{d \rho}{d t}\right)+4 \pi S\right\}\right],
\end{aligned}
$$

where $m$ is the mass absorption coefficient and $S$ is the source function.

Since the layer depth is small and the temperature gradient maintained is also small so that their product is very small in comparison with the temperature of the lower boundary, the temperature at an intermediate point may be approximately taken as the temperature of the lower boundary. The source function $S$ and the mass absorption coefficient $m$ may, therefore, be treated as independent of height in the equilibrium state. Then the linearized perturbation form of equation (12) may be obtained as

$$
\begin{aligned}
& 3 m^{2} \rho^{2} \frac{\partial \theta}{\partial t}-\frac{4 \pi m}{C_{P}}\left(S_{T}-\rho \alpha S_{P}\right) \nabla^{2} \theta-\frac{\partial}{\partial t} \nabla^{2} \theta \\
& =-\left(\beta+\frac{g}{C_{P}}\right)\left(3 m^{2} \rho^{2} w-\nabla^{2} w\right),
\end{aligned}
$$

where $S_{T}$ and $S_{\rho}$ denote respectively the partial derivatives of $S$ with respect to $T$ and $\rho$. 
We consider the case in which the boundaries are free as well as perfect conductors of both heat and solute. The density changes arise principally from thermal effects. The case of two free boundaries is most appropriate for stellar atmospheres as pointed by Spiegel [11]. The boundary conditions appropriate for the problem are

$$
w=\partial^{2} w / \partial z^{2}=\theta=\gamma=0 .
$$

\section{The Dispersion Relation}

We shall now analyze an arbitrary perturbation into complete set of normal modes by seeking solutions whose dependence on space and time coordinates is of the form

$\exp \left(i k_{x} x+i k_{y} y+n t\right) \sin k_{z} z$,

where $n$ is the growth rate and $k_{z}=s \pi / d, s$ being any integer and $d$ is the thickness of the layer and $k=\left(k_{x}^{2}+k_{y}^{2}+k_{z}^{2}\right)^{1 / 2}$ is the wave number of the perturbation.

Equations (8) - (10) and (14) give

$$
\begin{gathered}
\frac{\partial}{\partial t}\left(\nabla^{2} w\right)=g\left(\frac{\partial^{2}}{\partial x^{2}}+\frac{\partial^{2}}{\partial y^{2}}\right)\left(\alpha \theta-\alpha^{\prime} \gamma\right) \\
+v \nabla^{4} w \\
\left(\frac{\partial}{\partial t}-\kappa^{\prime} \nabla^{2}\right) \gamma=\beta^{\prime} w \\
\left(\frac{\partial}{\partial t}+E\right) \theta=-\left(\beta+\frac{g}{C_{P}}\right) w
\end{gathered}
$$

where

$$
E=\frac{4 \pi m\left(S_{T}-\rho \alpha S_{\rho}\right)}{C_{P}\left(1+\frac{3 m^{2} \rho^{2}}{k^{2}}\right)} .
$$

The system of equations (16) - (18) together with boundary conditions

$$
w=\partial^{2} w / \partial z^{2}=\theta=\gamma=0 .
$$

constitutes a characteristics value problem for $n$, for given values of the parameters and a given state of system is stable, neutral or unstable according as $n_{r}$, the real part of $\mathrm{n}$, is negative , zero or positive. Further, if $n_{r}=0 \Rightarrow n_{i}=0$ for all wave number $k$, then the principle of exchange of stabilities (PES) is valid otherwise we have overstability atleast when instability sets in as certain modes. The above system of equations can be solved by using linear stability analysis method.

Eliminating $\theta, \gamma$ from equations (16) - (18) and using (16), we obtain the dispersion relation

$$
\begin{aligned}
& n^{3}+\left[E+k^{2}\left(v+\kappa^{\prime}\right)\right] n^{2} \\
& +\left[k^{2} E\left(v+\kappa^{\prime}\right)+\Gamma\left(\beta+\frac{g}{C_{P}}\right)+v \kappa^{\prime} k^{4}\right. \\
& \left.+\Gamma^{\prime} \beta^{\prime}\right] n \\
& +\left[\Gamma\left(\beta+\frac{g}{C_{P}}\right) \kappa^{\prime} k^{2}+\left(v \kappa^{\prime} k^{4}+\Gamma^{\prime} \beta^{\prime}\right) E\right] \\
& =0
\end{aligned}
$$

where

$\Gamma=g \alpha \frac{k_{x}^{2}+k_{y}^{2}}{k^{2}}$ and $\Gamma^{\prime}=\frac{g \alpha^{\prime}\left(k_{x}^{2}+k_{y}^{2}\right)}{k^{2}}$.

\section{Discussion and Further Extensions}

Theorem 1: Acriterion that a stellar atmosphere in the presence of stable solute concentration gradient and radiative transfer effect is unstable if

$$
\begin{aligned}
& S_{T}-\frac{\rho}{T} S_{\rho}<0 \text { and }\left|\left(v \kappa^{\prime} k^{4}+\Gamma^{\prime} \beta^{\prime}\right) E\right| \\
& >\Gamma\left(\beta+\frac{g}{C_{P}}\right) \kappa^{\prime} k^{2} .
\end{aligned}
$$

Proof: Taking the dispersion relation (20), when

$$
\begin{aligned}
& S_{T}-\frac{\rho}{T} S_{\rho}<0 \text { and }\left|\left(\nu \kappa^{\prime} k^{4}+\Gamma^{\prime} \beta^{\prime}\right) E\right| \\
& >\Gamma\left(\beta+\frac{g}{C_{P}}\right) \kappa^{\prime} k^{2},
\end{aligned}
$$


The constant term in relation (20) is negative. Equation (20), therefore, involves one change of sign and hence contains one positive real root. The occurrence of positive root implies monotonic instability.

We thus obtain a criterion that a stellar atmosphere in the presence of stable solute concentration gradient and radiative transfer effect is unstable if

$$
\begin{aligned}
& S_{T}-\frac{\rho}{T} S_{\rho}<0 \text { and }\left|\left(v \kappa^{\prime} k^{4}+\Gamma^{\prime} \beta^{\prime}\right) E\right| \\
& >\Gamma\left(\beta+\frac{g}{C_{P}}\right) \kappa^{\prime} k^{2}
\end{aligned}
$$

Hence the result.

Further Extension-1: The rotation induces a component of vorticity in the direction of rotation. The effects of radiative transfer arise when self-absorption is not negligible. It may be of some interest to examine the influence of radiative transfer and rotation on thermosolutalconvective instability of a stellar atmosphere.

Theorem 2: The criterion for monotonic instability (22) also holds good in the presence of radiative transfer and rotation effects on thermosolutal-convective instability in Stern's type configuration in a stellar atmosphere.

Proof: Here we consider an infinite horizontal fluid layer heated from above and solute concentrated from below and acted on by a uniform rotation $\vec{\Omega}(0,0, \Omega)$ and gravity force $\vec{g}(0,0,-g)$. The linearized perturbed equations of motion become

$$
\begin{aligned}
\frac{\partial \vec{v}}{\partial t}=-\frac{1}{\rho_{0}} \nabla \delta p & +v \nabla^{2} \vec{v}-\vec{g}\left(\alpha \theta-\alpha^{\prime} \gamma\right) \\
& +2(\vec{v} \times \vec{\Omega}),
\end{aligned}
$$

Equations (9), (10) and (14) remain unaltered.

Equations (9), (10), (14) and (24) give

$$
\begin{gathered}
\frac{\partial}{\partial t}\left(\nabla^{2} w\right)=g\left(\frac{\partial^{2}}{\partial x^{2}}+\frac{\partial^{2}}{\partial y^{2}}\right)\left(\alpha \theta-\alpha^{\prime} \gamma\right) \\
+v \nabla^{4} w-2 \Omega \frac{\partial \zeta}{\partial z}
\end{gathered}
$$

$$
\frac{\partial \zeta}{\partial t}-v \nabla^{2} \zeta=2 \Omega \frac{\partial w}{\partial z},
$$

where

$$
\zeta=\frac{\partial v}{\partial z}-\frac{\partial u}{\partial y}
$$

denotes the z-component of vorticity, Equations (17) and (18) remain the same. Eliminating $\theta, \zeta$ and $\gamma$ from equations (17), (18), (25) and (26) and using expression (15), we obtain the dispersion relation

$$
\begin{aligned}
& n^{4}+\left[E+k^{2}\left(2 v+\kappa^{\prime}\right)\right] n^{3} \\
& +\left[v \kappa^{\prime} k^{4}+\Gamma^{\prime} \beta^{\prime}+E k^{2}\left(v+\kappa^{\prime}\right)+\Gamma\left(\beta+\frac{g}{C_{P}}\right)\right. \\
& \left.+v k^{2}\left(E+k^{2} \overline{v+\kappa^{\prime}}\right)+\frac{4 \Omega^{2} k_{z}^{2}}{k^{2}}\right] n^{2} \\
& +\left[\left(v \kappa^{\prime} k^{4}+\Gamma^{\prime} \beta^{\prime}\right) E+\Gamma\left(\beta+\frac{g}{C_{P}}\right) k^{2}\left(v+\kappa^{\prime}\right)\right. \\
& +v k^{2}\left\{v \kappa^{\prime} k^{4}+\Gamma^{\prime} \beta^{\prime}+k^{2} E\left(v+\kappa^{\prime}\right)\right\} \\
& \left.+\frac{4 \Omega^{2} k_{z}^{2}}{k^{2}}\left(E+\kappa^{\prime} k^{2}\right)\right] n \\
& +\left[v k^{2}\left\{\left(v \kappa^{\prime} k^{4}+\Gamma^{\prime} \beta^{\prime}\right) E+\kappa^{\prime} k^{2} \Gamma\left(\beta+\frac{g}{C_{P}}\right)\right\}\right. \\
& \left.+4 \Omega^{2} k_{z}^{2} \kappa^{\prime} E\right]=0 .
\end{aligned}
$$

When (22) is satisfied, the constant term in equation (27) is negative. The product of the roots must be negative. Therefore at least one root of equation (27) is positive and one root is negative. The occurrence of positive root implies monotonic instability. The criterion for monotonic instability thus holds good in the presence of radiative transfer and rotation effects on thermosolutal-convective instability in a stellar atmosphere.

Further Extension-2: Further we study the effect of collisions on thermosolutal-convective instability of a composite medium. Quite frequently it happens that the plasma is not fully ionized and may instead be permeated with neutral atoms. The plasma may be idealized, therefore, following Hans [6], as a composite mixture of a hydromagnetic (ionized) component and a neutral component, the two interacting through mutual collisional (frictional) effects. A partially ionized plasma represents a 
state which often exists in the universe. Hence it is of interest to investigate the influence of these frictional effects with neutrals on thermosolutalconvective instability of a composite medium.

Here we consider an infinite horizontal composite layer consisting of a finitely conducting hydromagnetic fluid of density $\rho$ and a neutral gas of density $\rho_{d}$, acted on by a uniform vertical magnetic field $\vec{H}(0,0, H)$ and gravity force $\vec{g}(0,0,-g)$. This layer is heated from above and solute concentrated from below such that a steady temperature gradient $\beta(=$ $d T / d z)$ and a concentration gradient $\beta^{\prime}(=$ $-d C / d z)$ are maintained. Regarding the model under consideration we observe that the number density of neutral particles is extremely smaller than the number density of ionized particles (Tanenbaum [12]). The pressure and buoyancy effects acting on neutral particles, therefore, have negligible influence (the volume fraction of the neutral particles being extremely small) whereas they are significant in the case of ionized component. In a partially ionized gas the magnetic field interacts with the charged component and is not directly affected by the neutral component (Parker [13]).

Now we prove the following Theorem :

Theorem 3: A criterion that the thermosolutalconvective instability of a composite stellar atmosphere in the presence of stable solute concentration gradient and collisional effects is unstable if

$$
\begin{aligned}
& D<0 \text { and }\left|\left(v \kappa^{\prime} k^{4}+\Gamma^{\prime} \beta^{\prime}\right) D\right| \\
& >\Gamma\left(\beta+\frac{g}{C_{P}}\right) \kappa^{\prime} k^{2} .
\end{aligned}
$$

Proof: Let $\vec{h}\left(h_{x}, h_{y}, h_{z}\right), \eta, \overrightarrow{v_{d}}$ and $v_{C}$ denote respectively the perturbation in magnetic field $\vec{H}$, the resistivity, the velocity of the neutral gas and the collision frequency between the two components of the composite medium. Then the linearized perturbation equations appropriate to the problem are

$$
\begin{aligned}
\frac{\partial \vec{v}}{\partial t}=-\frac{1}{\rho_{0}} \nabla \delta p & +v \nabla^{2} \vec{v}+\frac{1}{4 \pi \rho_{0}}(\nabla \times \vec{h}) \times \vec{H} \\
& +\vec{g} \frac{\delta \rho}{\rho_{0}}+\frac{\rho_{d} v_{C}}{\rho_{0}}\left(\overrightarrow{v_{d}}-\vec{v}\right),
\end{aligned}
$$

$\frac{\partial \overrightarrow{v_{d}}}{\partial t}=-v_{C}\left(\overrightarrow{v_{d}}-\vec{v}\right)$

$\frac{\partial \vec{h}}{\partial t}=\nabla \times(\vec{v} \times \vec{H})+\eta \nabla^{2} \vec{h}$,

$\nabla \cdot \vec{h}=0$,

together with equations (7), (9), (10) and (12). Equations (7), (9), (10), (12) and (28)-(31) give

$$
\begin{aligned}
& \begin{aligned}
\frac{\partial}{\partial t}\left(1+\frac{\alpha_{0} v_{C}}{\frac{\partial}{\partial t}+v_{C}}\right) & \left(\nabla^{2} w\right) \\
= & g\left(\frac{\partial^{2}}{\partial x^{2}}+\frac{\partial^{2}}{\partial y^{2}}\right. \\
& +v \nabla^{4} w+\frac{H}{4 \pi} \rho_{0} \\
\left(\frac{\partial}{\partial t}-\eta \nabla^{2}\right) h_{z} & =H \frac{\partial w}{\partial z},
\end{aligned} \\
& \left(\frac{\partial}{\partial t}+D\right) \theta=-\left(\beta+\frac{g}{C_{P}}\right) w, \\
& \left(\frac{\partial}{\partial t}-\kappa^{\prime} \nabla^{2}\right) \gamma=\beta^{\prime} w, \\
& \text { where } \alpha_{0}=\rho_{d} / \rho_{0}
\end{aligned}
$$$$
=g\left(\frac{\partial^{2}}{\partial x^{2}}+\frac{\partial^{2}}{\partial y^{2}}\right)\left(\alpha \theta-\alpha^{\prime} \gamma\right)
$$$$
+v \nabla^{4} w+\frac{H}{4 \pi \rho_{0}} \nabla^{2} \frac{\partial h_{z}}{\partial z},
$$

Eliminating $\theta, \gamma$ and $h_{z}$ from equations (32)(35) and using expression (15), we obtain the dispersion relation

$$
\begin{aligned}
& n^{5}+A_{4} n^{4}+A_{3} n^{3}+A_{2} n^{2}+A_{1} n+A_{0} \\
& =0
\end{aligned}
$$

where

$$
A_{4}=D+v_{C}\left(\alpha_{0}+1\right)+k^{2}\left(v+\eta+\kappa^{\prime}\right)
$$




$$
\begin{aligned}
A_{3}=\kappa^{\prime} \eta k^{4}+ & k^{2} D\left(\eta+\kappa^{\prime}\right) \\
& +\left(v_{C}+v k^{2}+\alpha_{0} v_{C}\right)\{D \\
& \left.+k^{2}\left(\eta+\kappa^{\prime}\right)\right\}+v k^{2} v_{C} \\
& +\Gamma\left(\beta+\frac{g}{C_{P}}\right)+\Gamma^{\prime} \beta^{\prime}+k_{z}^{2} V_{A}^{2}, \\
A_{2}=\kappa^{\prime} \eta k^{4} D+ & \left(v_{C}+v k^{2}+\alpha_{0} v_{C}\right)\left\{\kappa^{\prime} \eta k^{4}\right. \\
& \left.+k^{2} D\left(\eta+\kappa^{\prime}\right)\right\} \\
& +v k^{2} v_{C}\left\{D+k^{2}\left(\eta+\kappa^{\prime}\right)\right\} \\
& +\Gamma\left(\beta+\frac{g}{C_{P}}\right)\left(v_{C}+\eta k^{2}\right. \\
& \left.+\kappa^{\prime} k^{2}\right)+\Gamma^{\prime} \beta^{\prime}\left(v_{C}+\eta k^{2}+D\right) \\
+k_{z}^{2} & V_{A}^{2}\left(v_{C}+\kappa^{\prime} k^{2}+D\right), \\
A_{1}=\kappa^{\prime} \eta k^{4} D( & \left.+v k^{2}+\alpha_{0} v_{C}\right) \\
& +v k^{2} v_{C}\left\{\kappa^{\prime} \eta k^{4}\right. \\
& \left.+k^{2} D\left(\eta+\kappa^{\prime}\right)\right\} \\
& +\Gamma\left(\beta+\frac{g}{C_{P}}\right)\left\{\eta k^{2} v_{C}\right. \\
& \left.+\kappa^{\prime} k^{2}\left(v_{C}+\eta k^{2}\right)\right\} \\
& +\Gamma^{\prime} \beta^{\prime}\left\{\eta k^{2} v_{C}+D\left(v_{C}+\eta k^{2}\right)\right\} \\
& +k_{z}^{2} V_{A}^{2}\left\{\kappa^{\prime} k^{2} v_{C}\right. \\
& \left.+D\left(v_{C}+\kappa^{\prime} k^{2}\right)\right\},
\end{aligned}
$$

$A_{0}$

$=\eta k^{2} v_{C}\left\{\left(v \kappa^{\prime} k^{4}+\Gamma^{\prime} \beta^{\prime}\right) D+\Gamma\left(\beta+\frac{g}{C_{P}}\right) \kappa^{\prime} k^{2}\right\}$

$+k_{Z}^{2} V_{A}^{2} D \kappa^{\prime} k^{2} v_{C}$

where

$$
V_{A}^{2}=\frac{H^{2}}{4 \pi \rho_{0}}
$$

When

$$
\begin{aligned}
& \quad D<0 \text { and }\left|\left(v \kappa^{\prime} k^{4}+\Gamma^{\prime} \beta^{\prime}\right) D\right|> \\
& \Gamma\left(\beta+\frac{g}{C_{P}}\right) \kappa^{\prime} k^{2},
\end{aligned}
$$

the constant term in equation (36) is negative. Equation (36), therefore, involves one change of sign and hence contains one positive real root. The occurrence of positive root implies monotonic instability.

We thus obtain a criterion that the thermosolutal-convective instability of a composite stellar atmosphere in the presence of stable solute concentration gradient and collisional effects is unstable if

$$
\begin{aligned}
& D<0 \text { and }\left|\left(v \kappa^{\prime} k^{4}+\Gamma^{\prime} \beta^{\prime}\right) D\right| \\
& >\Gamma\left(\beta+\frac{g}{C_{P}}\right) \kappa^{\prime} k^{2} .
\end{aligned}
$$

The criterion for monotonic instability is the same in the presence or absence (Sharma and Sharma [14]) of the collisional effect on thermosolutal-convective instability of a composite stellar atmosphere.

\section{Acknowledgements}

The authors are grateful to all the three learned referees for their useful technical comments and valuable suggestions, which led to a significant improvement of the paper.

\section{References}

[1] R.J. Defouw, Thermal convective instability,Astrophys. J., Vol. 160, 1970, pp. 659-669.

[2] S. Chandrasekhar, Hydrodynamic and Hydromagnetic Stability, Oxford University Press, London, 1961.

[3] G. Veronis, On the finite amplitude instability in thermohaline convection, J. Marine Res., Vol. 23, 1965, pp. 1-17.

[4] D.A. Nield, The thermohaline convection with linear gradients,J. Fluid Mech., Vol. 29, 1967, pp. 545-558.

[5] R.C. Sharma andB. Singh, Thermosolutalconvective instability in a stellar atmosphere, $J$. Astrophys. Astronomy, Vol. 8, 1987, pp. 305314.

[6] H.K. Hans, Larmor radius and collisional effects on the combined Taylor and Kelvin instabilities in a composite medium, Nucl. Fusion, Vol. 8, 1968, pp. 89-92.

[7] A. Marcuand I. Ballai, Thermosolutal stability of a two-component rotating plasma with finite Larmor radius,Proc. Rom. Acad., Series A, Vol. 8(2), 2007, pp. 111-120. 
[8] C. Israel-Cookey and I. Amos, Soret and radiation absorption effects on the onset of magneto-thermosolutal convection in porous medium,J. Appl. Maths. Bioinform., Vol. 4(1), 2014, pp. 71-87.

[9] S.R. Sheri, O.A. Beg, P.Modugula and A. Kadir, Computation of transient radiative reactive thermosolutal magnetohydrodynamic convection in inclined MHD hall generator flow with dissipation and cross diffusion,Computational Thermal Sciences: An Int. J., Vol. 11 (6), 2019, pp. 541-563.

[10] W. Unno, andE.A. Spiegel, The eddigton approximation in the radiative heat equation,Publ. Astron. Soc. Japan, Vol. 18, 1966, pp. 85-95.

[11] E.A. Spiegel, Convective instability in a compressible atmosphere, Astrophys. J., Vol. 141, 1965, pp. 1068-1070.

[12] B.S. Tanenbaum, Plasma Phys. McGrawHill, New York, 1967.

[13] E.N. Parker, The solar-flare phenomenon and the theory of reconnection and annihilation of magnetic fields,Astrophys. J. Suppl., Vol. 8, 1963, pp. 177-211.

[14] R.C. Sharma andK.N. Sharma, Thermosolutal convective instability in stellar atmosphere,Acta Phys. Hungarica, Vol. 56, 1984, pp. 47-53.

\section{Creative Commons Attribution License 4.0 (Attribution 4.0 International, CC BY 4.0)}

This article is published under the terms of the Creative Commons Attribution License 4.0

https://creativecommons.org/licenses/by/4.0/deed.en_US 